\title{
COMPARAÇÃO DA MATÉRIA ORGÂNICA E DE OUTROS ATRIBUTOS DO SOLO ENTRE PLANTAÇÕES DE Acacia mangium E Eucalyptus grandis ${ }^{(1)}$
}

\author{
I. GARAY(2), A. KINDEL ${ }^{(3)}$, R. CARNEIRO(4), A. A. FRANCO(5), \\ E. BARROS(6) \& L. ABBADIE(7)
}

\begin{abstract}
RESUMO
Espécies de eucalipto e acácia são amplamente utilizadas em plantios agr oflorestais e reflorestamentos. Com o intuito de comparar a reconstituição das camadas orgânicas do solo, i.e., dos horizontes húmicos, estabelecendo-se uma relação com propriedades edáficas, sob plantações de Acacia mangium e E ucalyptus grandis, foram feitas coletas dos horizontes holorgânicos e hemiorgânicos do solo. Os referidos plantios encontravam-se na região de Tabuleiros Terciários no norte do estado do Espírito Santo e pertenciam à Reserva Natural da Vale do Rio Doce. As coletas foram feitas quando os plantios tinham sete anos de idade, em quatro estações. Acacia mangi um apresentou maior estoque de folhiço (10 t ha-1, em média), tanto na camada $L$, de folhas inteiras, como na camada $F$, de folhas fragmentadas, do que Eucal yptus grandis ( $5 \mathrm{t} \mathrm{ha}^{-1}$, em média). O material foliar em acácia apresentou menor relação $\mathrm{C} / \mathrm{N}$ que o de eucalipto, cerca da metade, decorrente dos maiores teores de nitrogênio. Quanto às análises químicas de carbono e nutrientes, no solo sob Acacia mangium, foram observadas, de modo geral, maiores quantidades destes elementos que no solo sob Eucalyptus grandis (e.g., carbono: 1,74 dag kg-1 vs 1,23 dag kg-1 e cálcio:

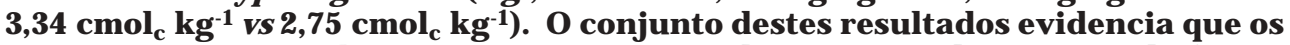
aportes orgânicos sob Acacia mangi um em relação a E ucalyptus grandis foram responsáveis pela maior incor poração de matéria orgânica e nutrientes ao solo. E stes dados, no entanto, comparados aos obti dos em estudos na floresta primária, mostraram que o carbono e os nutrientes do solo, em ambas as plantações, são menores que na floresta, evidenciando que, após sete anos de plantio, os teores de fertilidade e matéria orgânica do subhorizonte $A_{11}$ não estavam restabelecidos.
\end{abstract}

Termos de indexação: acácia, decomposição, eucalipto, serapilheira, relação C/N.

(1) Trabalho financiado pelo CNPq, Fundação J osé Bonifácio, FAPERJ e PROBIO. Recebido para publicação em janeiro de 2001 e aprovado em maio de 2003.

(2) Professora Adjunta do Departamento de Botânica da Universidade Federal do Rio deJ aneiro - UFRJ . Instituto de Biologia, CCS/ UFRJ , I lha do Fundão, CEP 21941-590 Rio de J aneiro (RJ ). E-mail: garay@biologia.ufrj.br

(3) Pesquisadora Associada da Embrapa Solos. Rua J ardim Botânico 1024, J ardim Botânico, CEP $22460-000$ Rio de J aneiro (RJ ). Bolsista da FAPERJ . E-mail: akindel@cnps.embrapa.br

(4) Engenheiro Florestal e Técnico do Departamento de Botânica, UFRJ .

(5) Pesquisador da Embrapa Agrobiologia. Rod. BR 465, Km 47, CEP 23851-970 Seropédica (RJ ). E-mail: avilio@cnpab.embrapa.br

(6) Pesquisadora do Instituto Nacional de Pesquisas da Amazônia - INPA. Av. AndréAraújo 2936, Petrópolis, CEP 69083-000 Manaus (AM). E-mail: ebarros@inpa.gov.br

(7) Pesquisador École Normal Supérieure - ENS. Laboratoire d'Ecologie, ENS, 46, rue d'UIm, 75005, Paris, França. E-mail: abbadie@biologie.ens.fr 


\title{
SUMMARY: COMPARISON OF ORGANIC MATTER AND OTHER SOIL PROPERTIES IN Acacia mangium AND Eucalyptus grandis PLANTATIONS
}

\begin{abstract}
Eucalyptus and Acacia species are widely used in agrosilviculture and reforestry practices. I n order to evaluatethereconstitution of organic soil layers - i.e. thehumic horizons -and establish relationships to thesoil properties, thehol organic and hemiorganichorizons weresampled under Acacia mangium and Eucalyptus grandis plantations. Theseplantations lie in the Tertiary Tableland region in the North of Espírito Santo State, Brazil, and are part of the Natural Reservation Area of the Vale do Rio Doce Company. Samples were collected in seven-year-old plantations in four seasons. Acacia mangium presented a higher litter stock (mean of $10 \mathrm{tha}^{-1}$ ) in both the $\mathrm{L}$ (undecomposed leaves) and the $\mathrm{F}$ layer (fragmented leaves) than Eucalyptus grandis (mean of $5 \mathrm{t} \mathrm{ha}^{-1}$ ). Thefoliar material of Acacia al so presented smaller $\mathrm{C} / \mathrm{N}$ ratios than Eucalyptus, al most half as small, due to higher nitrogen contents. In regard to the chemical analyses of carbon and nutrients, higher quantities of the elements were observed in the soil under Acacia mangium than under Eucalyptus grandis (e.g., carbon: 1.74 vs $1.23 \mathrm{dag} \mathrm{kg}^{-1}$ and calcium: 3.34 vs $2.75 \mathrm{cmol}_{\mathrm{c}} \mathrm{kg}^{-1}$ ). This set of results suggests that theorganic contri bution of Acacia mangi um was responsible for higher organic matter and nutrient inputs in thesoil than Eucalyptus grandis. However, when these data were compared to those found in studies of primary forest, carbon and nutrient soil contents in both plantations were smaller than in the forest, indicating that after seven years fertility and organic matter of the $A_{11}$ horizon had not been reestabli shed in the plantations.
\end{abstract}

Index terms: acacia, C/ N ratio, decomposition, eucalyptus, litter.

\section{NTRODUÇÃO}

Considerando ter sido mais de $90 \%$ da cobertura vegetal da Mata Atlântica já devastada, o plantio tanto de espécies nativas como de espécies agroflorestais é importante não só para garantir a recuperação deáreas degradadas, mas também para contribuir com um estoque madeireiro alternativo. Espécies de eucal ipto têm si do empregadas em larga escala em reflorestamentos e agrossilviculturas desde o início do século passado; todavia, sua expansãotem sidoimpulsionada a partir de meados da década de 60 com o estabelecimento do programa deincentivos fiscais, que visava à sua utilização como matéria-prima nas indústrias, sobretudo para obter celulose e energia (Lima, 1987). O rápido crescimento desta árvore, sua adaptação às mais variadas condições edafoclimáticas e a ampla gama de sua utilização explicam o sucesso obtido por esta espécie.

Segundo levantamento feito por Higa \& Higa (2000), espécies de acácia também vêm sendo empregadas para o estabelecimento de plantações florestais. O interesse por leguminosas arbóreas é despertado por seu poder de fixação de nitrogênio atmosférico e a simbiose com fungos micorrízicos, o que facilita seu estabel ecimento em sol os pobres em nutrientes e matéria orgânica (Franco et al., 1995). Como a fertilidade dos solos tropicais depende fortemente da qualidade e quantidade dos estoques orgânicos aportados ao solo, o conhecimento da reconstituição das camadas orgânicas do solo ou horizontes húmicos, relacionados com propriedades edáficas, traz informações relevantes para o futuro manejo destes plantios arbóreos (Garay \& Kindel, 2001; Kindel \& Garay, 2002).

Nesta perspectiva, avaliaram-se as diferenças dos sol os sob uma plantação de Acacia mangium eoutra de Eucalyptus grandis em região de Tabuleiros Terciários no norte do Espírito Santo. Estes resultados foram confrontados aos observados em estudos feitos na floresta primária que predomina na região, a Mata Atlântica de Tabuleiros, e que se encontra sob as mesmas condi ções cl imáticas e sobre o mesmo tipo de solo que as plantações (Kindel et al., 1999; Kindel \& Garay, 2001). Desta forma, o efeito de cada um destes plantios na matéria orgânica e outros atributos do sol o poderá ser mais bem compreendido.

\section{MATERIAL E MÉTODOS}

\section{Caracterização das áreas de estudo}

As plantações de Acacia mangium Wild e Eucalyptus grandis Hill ex Maid estavam instaladas na Reserva Natural da Vale do Rio Doce, localizada entre os municípios de Linhares e São Mateus, no 
norte do estado do Espírito Santo. O relevo desta regiãoé caracterizado por uma seqüência de colinas tabulares, formadas por sedimentos terciários, eque atingem, em média, 70-80 m dealtitude (Meis, 1976). O clima da reserva apresenta estação chuvosa no verão e seca no inverno, sendo a preci pitação média anual de $1.100 \mathrm{~mm}$, enquanto a temperatura média anual é de ${ }^{\circ} 3^{\circ} \mathrm{C}$, média máxima e mínima de 34 e $15^{\circ} \mathrm{C}$, respectivamente; e a umidade relativa do ar de $83 \%$. A vegetação do entorno corresponde à Floresta Atlântica de Tabuleiros e apresenta características únicas que a distinguem da F loresta Atlântica típica, istoé, a que recobrea região serrana (Rizzini et al., 1997). O solo predominante é o Argissolo Amarelo, ocupando as áreas em relevo plano ou suave ondulado. Este solo apresenta um horizonte A moderado, que alcança $17 \mathrm{~cm}$, e um $B$ textural, que atinge quase $2 \mathrm{~m}$, sendo bem drenado. A capacidade de troca catiônica, assim como a soma de bases, é baixa, caracterizando a pobreza química deste solo (Garay et al., 1995b).

A plantação de A. mangium era constituída de diversos tal hões com espaçamentos distintos, dentre os quais três parcelas de 21 × 21 m com 49 árvores em cada parcela, separadas as árvores $3 \mathrm{~m}$ uma da outra. A plantação de E. grandis era constituída de uma parcela única de 1 ha, enquanto o espaçamento entre as árvores era de 3,0 × 2,7 m. As covas para o plantio de A. mangium foram tratadas com superfosfato simples ( $225 \mathrm{~g}$ por cova) eas covas para o plantio de E. grandis com $120 \mathrm{~g}$ de superfosfato simples e $50 \mathrm{~g}$ de $25-00-25$ de NPK em cobertura (a fonte de $\mathrm{N}$ do formulado é a uréia e a fonte de $\mathrm{K}$ é $\mathrm{O}$ $\mathrm{KCl}), 180$ dias após o plantio.

\section{Amostragem}

Em cada uma das parcelas de A. mangium, as árvores foram numeradas de 1 a 49 e usadas, como referência, para a amostragem aleatória. As amostragens na parcela de E. grandis foram feitas ao longo de um transecto, a intervalos aleatórios, com no mínimo uma distância de 5 m entre os pontos amostrais. O método empregado foi o mesmo que para o estudo das formas de húmus, descrito em Kindel \& Garay (2001; 2002). As amostragens foram feitas em agosto e novembro de 1991 e fevereiro e junho de 1992, em um total de 13 amostras por estação, e consistiam da coleta dos horizontes holorgânicos e hemiorgânicos. Os horizontes hol orgânicos, camada L e camada $F$, foram col etados dentro de um quadrado de $25 \times 25 \mathrm{~cm}$. Os horizontes hemiorgânicos, subdivididos de 0 a $4-6 \mathrm{~cm}$ (ou subhorizonte $A_{11}$ ) e de 4-6 a $14-16 \mathrm{~cm}$ (ou subhorizonte $A_{12}$ ) de profundidade, foram col etados, respectivamente, no mesmo quadrado e em um cilindro de $10 \mathrm{~cm}$ de diâmetro por $10 \mathrm{~cm}$ de altura. A espessura do subhorizonte $A_{11}$ foi determinada de acordo com o arado utilizado no preparo da terra para o plantio.

\section{Análise dos horizontes holorgânicos}

A separação dos horizontes holorgânicos foi feita de acordo com as características macromorfológicas (Babel, 1975; Garay et al., 1995a,b), sendo diferenciados da seguinte forma: camada $L$, as folhas são recém-caídas e não fragmentadas; camada F, o fol hiçoécomposto de fragmentos defácil identificação, e a camada apresenta pequenas quantidades de material orgânico fino menor que $2 \mathrm{~mm}$. Este material foi seco em estufa a $60^{\circ} \mathrm{C}$ e pesado. A percentagem de matéria orgânica de cada horizonte foi estimada, queimando-se, em mufla a $550^{\circ} \mathrm{C}$, por quatro horas, de 0,2 a 0,4 g de material foliar moído. Os teores de carbono enitrogênio foram determinados em aparelhos denominados, respectivamente, de CARMOGRAF e TECATOR Kjeltec Auto 1030 Analyzer. Para estas análises, foram preparadas amostras compostas, a partir de três subamostras.

\section{Análise dos horizontes hemiorgânicos}

As amostras dos horizontes hemi orgânicos foram secas e peneiradas no laboratório. A fração inferior a $2 \mathrm{~mm}$ dos suhorizontes $A_{11}$ e $A_{12}$ foi enviada para a EMBRAPA-Agrobiologia (Seropédica, RJ .), onde foram realizadas as análises químicas de carbono, nitrogênio e fertilidade (EMBRAPA, 1979).

\section{Análise estatística}

Para testar a significância entre as duas plantações, utilizou-se o teste t de Student após normalização dos dados pela transformação " $x=\sqrt{ } x$ ". Quando o " $n$ " amostral era pequeno, como no caso das amostras compostas de folhiço para a determinação da percentagem de matéria orgânica e do teor de nitrogênio, fazia-se o uso do teste nãoparamétrico U de Mann-Whitney. A correlação nãoparamétrica de Pearson ( $r$ ), que testa uma relação linear entre dois conjuntos de dados, foi feita para os atributos do horizonteA com a ajuda do programa Minitab. A significância do $r$ foi calculada pela distribuiçãonormal demédia $Z=1 / 2 \ln (1+r / 1-r)$ e variância $\mathrm{S}^{2}=\mathrm{N}-3$.

\section{RESULTADOS E DISCUSSÃO}

\section{Análise dos horizontes holorgânicos}

Verificou-se que a plantação de A. mangium acumulou mais fol hiço do que a plantação de $E$. grandis, tanto na camada $\mathrm{L}$ como na camada $\mathrm{F}$ (Figura 1). Considerando o total acumulado, as diferenças entreas plantações foram mais acentuadas nos meses de novembro (16,4 vs $5,9 \mathrm{t} \mathrm{ha}^{-1}$ ) e agosto ( 12,0 vs 6,0 t ha- $^{-1}$ ) e menos acentuadas em junho (6,9 vs 3,9 t ha $^{-1}$ ) efevereiro $\left(6,3\right.$ vs 3,6 t ha $\left.^{-1}\right)$. Os maiores estoques observados em ambas as plantações coincidem com o período de queda foliar em uma área de Mata Atlântica de Tabuleiros vizinha 
(Louzada et al., 1997). Segundo esseautor, a fenologia dos aportes está relacionada com a variação da preci pitação ao longo do ano, ocorrendo maior queda dematerial nofim do inverno regional, notadamente em outubro, tal como nas plantações. O acúmulo de fol hiço nas camadas holorgânicas em um trecho primário e dois secundários da Mata de Tabuleiros foi, em média, de 4,0 e 6,0 t ha-1, respectivamente (Kindel et al., 1999; Kindel \& Garay, 2001). Em uma plantação de Coffea robusta Linden com 10 anos, também localizada nesta região, o estoque de fol hiço variou de 3,3 a 6,5 t ha-1 (Pellens \& Garay, 1999). A plantação de $A$. mangium é, portanto, a que mais acumulou matéria orgânica no solo.

O acúmulo pode ser decorrente da baixa vel oci dade de decomposição e, ou, do grande aporte orgânico. A vel ocidade de decomposição é regulada por diversos fatores, dentreel es a qualidade química do material aportado, que pode ser expressa pela relação C/N (Swift et al., 1979): ou seja, baixos teores de nitrogênio estão relacionados com uma menor velocidade de decomposição. O teor de nitrogênio das fol has pouco decompostas e que constituem a

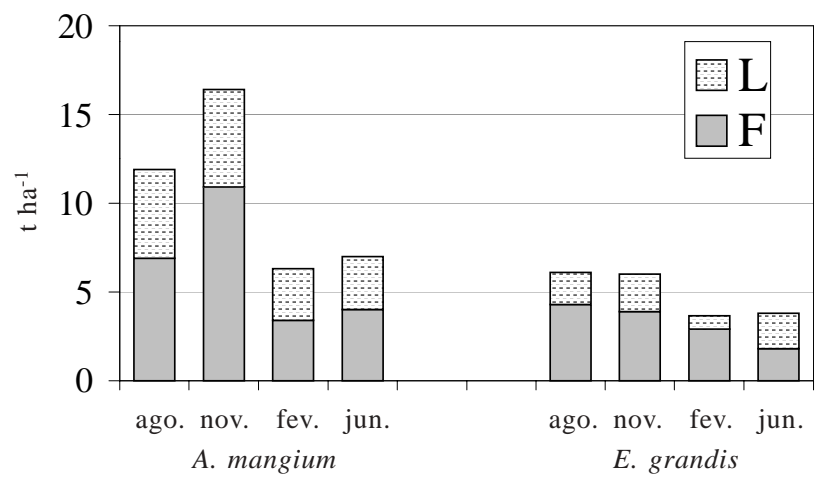

Figura 1. Estoque de matéria orgânica nas camadas holorgânicas em diferentes estádios de decomposição. L: camada de folhas inteiras e F : camada de fol has fragmentadas mais material fino menor que $2 \mathrm{~mm}$. $n=13$. ago: agosto; nov: novembro; fev: fevereiro e jun: junho. camada $L$ em $A$. mangium foi quase o dobro que em E. grandis, indicando que a qualidade do material foliar da primeira é melhor (Quadro 1). Este valor para A. mangium (1,56 dag kg-1) está dentro dos limites observados para a Mata de Tabuleiros: 1,75 dag $\mathrm{kg}^{-1}$ na Mata Alta, a mata primária, 1,27 dag kg-1 na Mata de Córrego e 1,37 dag kg-1 em uma capoeira que se desenvolveu após corte e queima da vegetação (Kindel \& Garay, 2001). O teor de nitrogênio medido nas folhas de $E$. grandis $\left(0,86\right.$ dag $\mathrm{kg}^{-1}$, Quadro 1) foi menor que os supramencionados, estando mais próximo aos obtidos por Kindel \& Garay (2002) em ecossistemas

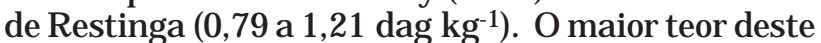
nutriente em acácia que em eucalipto mantém-se na camada de fol has fragmentadas $F$ e em sua fração fina.

Existe uma relação entre as altas relações $\mathrm{C} / \mathrm{N}$, ou baixos teores de nitrogênio, eo acúmulo orgânico e a presença de um Moder (forma de húmus caracterizada pela coexistência de várias camadas em diferentes estádios de decomposição) (Berthelin et al., 1994; Garay et al., 1995a,b; Kindel \& Garay, 2002). Parece, portanto, que oacúmul o observadoem A. mangium em comparação $\mathrm{E}$. grandis éo resultado de uma mai or queda defol has e não de uma limitação do teor de nitrogênio e, conseqüentemente, de uma menor velocidade de decomposição. De fato, um aporte foliar anual de $7,9 \mathrm{tha}^{-1}$ foi medido por Bernhard-Reversat (1993) em uma plantação de A. mangi um no Congo, enquanto o de eucalipto foi de apenas 4,9 $\mathrm{t} \mathrm{ha}^{-1}$. Da mesma forma, a pobreza em nitrogênio do fol hiço de eucal ipto em rel ação à acácia foi verificada por esse autor.

Confrontando a média dos meses de maior acúmulo (agosto e novembro) com os de menor (fevereiro e junho), verificou-se que, no plantio de acácia, 7,5 t ha-1 de matéria orgânica foram incorporadas ao solo por meio da decomposição, enquanto, em eucalipto, apenas $2,2 \mathrm{t} \mathrm{ha}^{-1}$. A plantação de A. mangium contribuiu, portanto, com um grande estoque de matéria orgânica para o solo. A estimativa do estoque de nitrogênio das camadas hol orgânicas foi de 162 e $42 \mathrm{~kg} \mathrm{ha}^{-1}$ em A. mangium e E. grandis,

Quadro 1. Teor de carbono orgânico e de nitrogênio e valores da relação C/N das camadas holorgânicas

\begin{tabular}{|c|c|c|c|c|c|c|}
\hline \multirow{2}{*}{ Camada } & \multicolumn{2}{|c|}{ Carbono orgânico } & \multicolumn{2}{|c|}{ Nitrogênio } & \multicolumn{2}{|c|}{$\mathbf{C} / \mathrm{N}$} \\
\hline & A. mangi um & E. grandis & A. mangium & E. grandis & A. mangium & E. grandis \\
\hline & \multicolumn{4}{|c|}{ dag kg-1 $^{-1}$} & & \\
\hline L (folhas inteiras) & $47,0(0,2)^{(1)}$ & $48,5^{*}(0,1)$ & $1,56^{*}(0,08)$ & $0,86(0,02)$ & $30,3(1,4)$ & $56,4^{*}(0,9)$ \\
\hline F (fragmentos) & $39,4(3,3)$ & $47,2^{*}(0,3)$ & $1,69 *(0,10)$ & $0,98(0,03)$ & $23,5(1,8)$ & $48,4^{*}(1,5)$ \\
\hline F (fração fina) & $19,9(2,0)$ & $15,1(0,3)$ & $1,08^{*}(0,11)$ & $0,50(0,01)$ & $18,5(0,3)$ & $30,6 *(0,3)$ \\
\hline
\end{tabular}

(1) Os números correspondem à média $\pm($ erro $(s / / n))$. Significância pelo teste $U$ entre as plantações.

*: Significativo a $5 \%$. $n=3$. O teste aparece na coluna com o maior valor. 
respectivamente. Este dado para acácia foi similar aos calculados por Gama-Rodrigues et al. (1999) em alguns plantios de espécies nativas do sudeste da Bahia. Para eucalipto, no entanto, o estoque de nitrogênio contido na serapilheira ficou bem abaixo dos verificados por esses autores.

\section{Análise dos horizontes hemiorgânicos}

Comparação entre as plantações: Considerando a pouca variabilidade estacional, os dados foram agrupados em uma média (Quadro 2). As concentrações de C, N, P e a soma de bases (SB) foram sempre maiores no subhorizonte $A_{11}$ da plantação de A. mangium que no da de E. grandis, excetuando-se o cál cio e o magnésio trocáveis e a SB no subhorizonte $A_{12}$. O cál cio é a base que al cança as mai ores concentrações, o queé observado também no solo da Floresta de Tabuleiro, embora, nesta floresta, seus valores estejam próximos de $8,84 \mathrm{cmol}_{\mathrm{c}} \mathrm{kg}^{-1}$ (Kindel et al., 1999; Kindel \& Garay, 2001), enquanto, nas plantações, não ultrapassam $3,34 \mathrm{cmol}_{\mathrm{C}} \mathrm{kg}^{-1}$. Sódio e potássio tiveram as menores concentrações, e magnésio intermediárias, também como na área de floresta. Este padrão ( $\mathrm{Ca}>\mathrm{Mg}>$ $\mathrm{K}>\mathrm{Na}$ ) corresponde ao observado para a análise química da serapilheira daquelas diferentes coberturas agroflorestais em região detabuleiros na Bahia (Gama-Rodrigues et al., 1999).

Estes fatos demonstram a íntima relação da vegetação com o solo. Na região de estudo, o sol o é, quimicamente, muito pobre, sendo, portanto, a maior parte dos nutrientes proveniente da vegetação: nota- secomo os nutrientes estão bem correlacionados com oteor de C do horizonteA (Quadro 3). Sendo assim, a mai or soma de bases encontrada no subhorizonte $A_{11}$ de $A$. mangium que no de $E$. grandis está relacionada, como mencionado, com a maior quantidade de matéria orgânica observada tantono horizonte hol orgânico como no hemiorgânico nesta plantação (Quadro 2 e 3).

O teor de C está relacionado com os valores de capadidade detroca catiônica (CTC), muito importante para a manutenção da fertilidade deste Argissolo Amarelo (Quadro 3). As percentagens de saturação por bases (V) foram maiores em E. grandis que em A. mangium, em virtude da menor CTC em E. grandis, decorrente do menor teor de carbono observado no solo do eucaliptal. Esta maior percentagem de saturação por bases também condiciona o pH mais al to observado em E. grandis (Quadro 3).

Comparação das plantações com a floresta: Trabalhando em florestas primárias e interferidas da região de Tabuleiros, Garay et al. (1995a,b), Kindel et al. (1999) e Kindel \& Garay (2001; 2002) encontraram um gradiente nas quantidades de carbono e nutrientes nos horizontes hemiorgânicos: mai ores teores destes el ementos foram encontrados no subhorizonte $A_{11}$ (Figura 2). Ficou demonstrado que os teores de $C$ eram de quatro a cinco vezes maiores no subhorizonte $A_{11}$ que no subhorizonte $A_{12}$ da floresta primária (e.g., 3,71 dag kg-1 no $A_{11}$

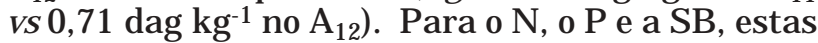
diferenças eram de duas a quase seis vezes entre os subhorizontes (e.g., $\mathrm{P}: 14,3 \mathrm{mg} \mathrm{kg}^{-1}$ no $\mathrm{A}_{11}$ vs $2,5 \mathrm{mg} \mathrm{kg}^{-1}$ no $\left.\mathrm{A}_{12}\right)$.

Quadro 2. Características químicas da fração fina do subhorizonte $A_{11}$ e subhorizonte $A_{12}$

\begin{tabular}{|c|c|c|c|c|c|c|c|c|c|c|c|c|}
\hline $\begin{array}{l}\text { Sub- } \\
\text { horiz. }\end{array}$ & $\mathbf{C}$ & $\mathbf{N}$ & $\mathbf{P}$ & $\mathrm{Ca}^{2+}$ & $\mathbf{M g}^{2+}$ & $\mathrm{Na}^{+}$ & $\mathbf{K}^{+}$ & SB & СТC & $\mathbf{v}$ & $\mathbf{C} / \mathbf{N}$ & $\mathrm{pH}\left(\mathrm{H}_{2} \mathrm{O}\right)$ \\
\hline & \multicolumn{2}{|c|}{ _ dag kg-1__ } & $\mathrm{mg} \mathrm{kg}^{-1}$ & \multicolumn{6}{|c|}{$-\mathrm{cmol}_{\mathrm{c}} \mathrm{kg}^{-1}$} & $\%$ & & \\
\hline \multicolumn{13}{|c|}{ Acacia mangium } \\
\hline$A_{11}$ & $\begin{array}{c}1,74 \\
(0,05)^{(1)}\end{array}$ & $\begin{array}{c}0,15 \\
(0,00)\end{array}$ & $\begin{array}{c}3,70 \\
(0,41)\end{array}$ & $\begin{array}{c}3,34 \\
(0,13)\end{array}$ & $\begin{array}{l}1,50 \\
(0,04)\end{array}$ & $\begin{array}{c}0,05 \\
(0,00)\end{array}$ & $\begin{array}{c}0,18 \\
(0,01)\end{array}$ & $\begin{array}{c}5,07 \\
(0,16)\end{array}$ & $\begin{array}{c}9,59 \\
(0,22)\end{array}$ & $\begin{array}{c}52,6 \\
(0,91)\end{array}$ & $\begin{array}{c}11,8 \\
(0,18)\end{array}$ & $\begin{array}{c}4,89 \\
(0,04)\end{array}$ \\
\hline$A_{12}$ & $\begin{array}{c}1,19 \\
(0,02)\end{array}$ & $\begin{array}{c}0,11 \\
(0,00)\end{array}$ & $\begin{array}{c}2,58 \\
(0,27)\end{array}$ & $\begin{array}{c}1,77 \\
(0,14)\end{array}$ & $\begin{array}{c}0,90 \\
(0,05)\end{array}$ & $\begin{array}{c}0,05 \\
(0,00)\end{array}$ & $\begin{array}{c}0,13 \\
(0,01)\end{array}$ & $\begin{array}{c}2,85 \\
(0,18)\end{array}$ & $\begin{array}{c}6,83 \\
(0,18)\end{array}$ & $\begin{array}{c}40,5 \\
(1,76)\end{array}$ & $\begin{array}{c}11,3 \\
(0,25)\end{array}$ & $\begin{array}{c}4,61 \\
(0,07)\end{array}$ \\
\hline \multicolumn{13}{|c|}{ Eucalyptus grandis } \\
\hline$A_{12}$ & $\begin{array}{c}1,23 \\
(0,04) \\
0,76 \\
(0,03)\end{array}$ & $\begin{array}{c}0,10 \\
(0,00) \\
0,08 \\
(0,00)\end{array}$ & $\begin{array}{c}1,86 \\
(0,09) \\
1,80 \\
(0,09)\end{array}$ & $\begin{array}{c}2,75 \\
(0,15) \\
1,75 \\
(0,15)\end{array}$ & $\begin{array}{c}1,25 \\
(0,05) \\
0,77 \\
(0,05)\end{array}$ & $\begin{array}{c}0,04 \\
(0,00) \\
0,04 \\
(0,00)\end{array}$ & $\begin{array}{c}0,11 \\
(0,00) \\
0,08 \\
(0,00)\end{array}$ & $\begin{array}{c}4,15 \\
(0,19) \\
2,64 \\
(0,19)\end{array}$ & $\begin{array}{c}6,76 \\
(0,22) \\
5,04 \\
(0,22)\end{array}$ & $\begin{array}{c}60,0 \\
(1,39) \\
50,1 \\
(1,89)\end{array}$ & $\begin{array}{c}13,8 \\
(1,25) \\
9,85 \\
(0,31)\end{array}$ & $\begin{array}{c}5,39 \\
(0,04) \\
5,08 \\
(0,06)\end{array}$ \\
\hline \multicolumn{13}{|c|}{ Teste t entre as plantações(2) } \\
\hline$A_{11}$ & $* * *$ & $* * *$ & $* * *$ & $* *$ & $* * *$ & $* *$ & $* * *$ & $* * *$ & $* * *$ & $* * *$ & ns & $* * *$ \\
\hline$A_{12}$ & $* * *$ & $* * *$ & $* *$ & ns & ns & $* *$ & $* * *$ & ns & $* * *$ & $* * *$ & $* * *$ & $* * *$ \\
\hline
\end{tabular}

(1) Os números correspondem à média $\pm($ erro $(\mathrm{s} / \mathrm{Vn}))$ dos dados col etados nas quatro estações. ${ }^{\text {(2) }}$ Significância pel o teste t de Student para A. mangium vs E. grandis. SB: soma de bases; CTC: capacidade de troca catiônica; V: índice de saturação por bases. $\mathrm{n}=52$. ns: Não-signficativo a $5 \%$ *, ** e ***: Significativos a $5,1,0,1 \%$, respectivamente. 
Quadro 3. Correlação de Pearson (r) entre as propriedades edáficas do horizonte A

\begin{tabular}{|c|c|c|c|c|c|c|c|c|c|c|c|}
\hline & C & $\mathbf{N}$ & $\mathbf{P}$ & $\mathrm{Ca}^{2+}$ & $\mathbf{M g}^{2+}$ & $\mathrm{Na}^{+}$ & $\mathbf{K}^{+}$ & SB & СТC & v & $\mathbf{C} / \mathbf{N}$ \\
\hline \multicolumn{12}{|l|}{ C } \\
\hline$N$ & $\begin{array}{l}0,86 \\
* * * * *\end{array}$ & & & & & & & & & & \\
\hline$P$ & $\begin{array}{r}0,28 \\
* * * *\end{array}$ & $\begin{array}{l}0,33 \\
* * * *\end{array}$ & & & & & & & & & \\
\hline $\mathrm{Ca}^{2+}$ & $\begin{array}{r}0,69 \\
* * * * *\end{array}$ & $\begin{array}{l}0,65 \\
* * * *\end{array}$ & $\underset{* * *}{0,23}$ & & & & & & & & \\
\hline $\mathrm{Mg}^{2+}$ & $\begin{array}{r}0,69 \\
* * * *\end{array}$ & $\begin{array}{l}0,58 \\
* * * *\end{array}$ & 0,18 & $\begin{array}{l}0,80 \\
* * * *\end{array}$ & & & & & & & \\
\hline $\mathrm{Na}^{+}$ & $\begin{array}{r}0,45 \\
* * * * *\end{array}$ & $\begin{array}{l}0,39 \\
* * * *\end{array}$ & $\begin{array}{c}-0,11 \\
n s\end{array}$ & $\begin{array}{l}0,33 \\
* * * *\end{array}$ & $\begin{array}{l}0,34 \\
* * * *\end{array}$ & & & & & & \\
\hline $\mathrm{K}^{+}$ & $\begin{array}{l}0,47 \\
* * * *\end{array}$ & $\begin{array}{l}0,45 \\
* * * *\end{array}$ & $\begin{array}{l}0,27 \\
* * * *\end{array}$ & $\begin{array}{l}0,47 \\
* * * *\end{array}$ & $\begin{array}{l}0,43 \\
* * * *\end{array}$ & $\begin{array}{l}0,23 \\
* * *\end{array}$ & & & & & \\
\hline SB & $\begin{array}{r}0,73 \\
* * * * *\end{array}$ & $\begin{array}{l}0,66 \\
* * * *\end{array}$ & $\underset{* * *}{0,23}$ & $\begin{array}{l}0,98 \\
* * * *\end{array}$ & $\begin{array}{l}0,88 \\
* * * *\end{array}$ & $\begin{array}{l}0,36 \\
* * * *\end{array}$ & $\begin{array}{l}0,51 \\
* * * *\end{array}$ & & & & \\
\hline СТС & $\begin{array}{r}0,89 \\
* * * *\end{array}$ & $\begin{array}{l}0,82 \\
* * * *\end{array}$ & $\begin{array}{l}0,29 \\
* * * *\end{array}$ & $\begin{array}{l}0,82 \\
* * * *\end{array}$ & $\begin{array}{l}0,80 \\
* * * *\end{array}$ & $\begin{array}{l}0,48 \\
* * * *\end{array}$ & $\begin{array}{r}0,52 \\
* * * *\end{array}$ & $\begin{array}{r}0,86 \\
* * * *\end{array}$ & & & \\
\hline v & $\begin{array}{c}0,22 \\
* *\end{array}$ & $\underset{* *}{0,18}$ & 0,05 & $\begin{array}{l}0,75 \\
* * * *\end{array}$ & $\begin{array}{l}0,65 \\
* * * * *\end{array}$ & 0,08 & $\begin{array}{c}0,24 \\
* * *\end{array}$ & $\begin{array}{r}0,75 \\
* * * *\end{array}$ & $\begin{array}{r}0,32 \\
* * * *\end{array}$ & & \\
\hline $\mathrm{C} / \mathrm{N}$ & $\begin{array}{c}0,19 \\
* *\end{array}$ & $\begin{array}{c}-0,20 \\
* *\end{array}$ & $\begin{array}{c}-0,01 \\
\text { ns }\end{array}$ & $\begin{array}{c}0,13 \\
\text { ns }\end{array}$ & $\begin{array}{l}0,25 \\
* * *\end{array}$ & $\begin{array}{c}0,03 \\
\text { ns }\end{array}$ & $\begin{array}{c}0,03 \\
\text { ns }\end{array}$ & $\begin{array}{c}0,17 \\
*\end{array}$ & $\underset{*}{0,14}$ & $\underset{*}{0,15}$ & \\
\hline $\mathrm{pH}$ & $\begin{array}{c}0,01 \\
\text { ns }\end{array}$ & $\begin{array}{c}-0,03 \\
\text { ns }\end{array}$ & $\begin{array}{c}-0,07 \\
\text { ns }\end{array}$ & $\begin{array}{r}0,55 \\
* * * *\end{array}$ & $\begin{array}{l}0,43 \\
* * * * *\end{array}$ & $\begin{array}{c}0,02 \\
\text { ns }\end{array}$ & $\underset{*}{0,14}$ & $\begin{array}{l}0,53 \\
* * * *\end{array}$ & $\begin{array}{c}0,10 \\
\text { ns }\end{array}$ & $\begin{array}{l}0,84 \\
* * * *\end{array}$ & $\begin{array}{c}0,13 \\
\text { ns }\end{array}$ \\
\hline
\end{tabular}

(1) Significância do $r$ dada pela distribuição normal: $\mathrm{n}=205 . \mathrm{ns}$ : Não-signficativo a $5 \%$. ***, *** e ****: Significativos a 5, 1, 0,1, $0,01 \%$, respectivamente.
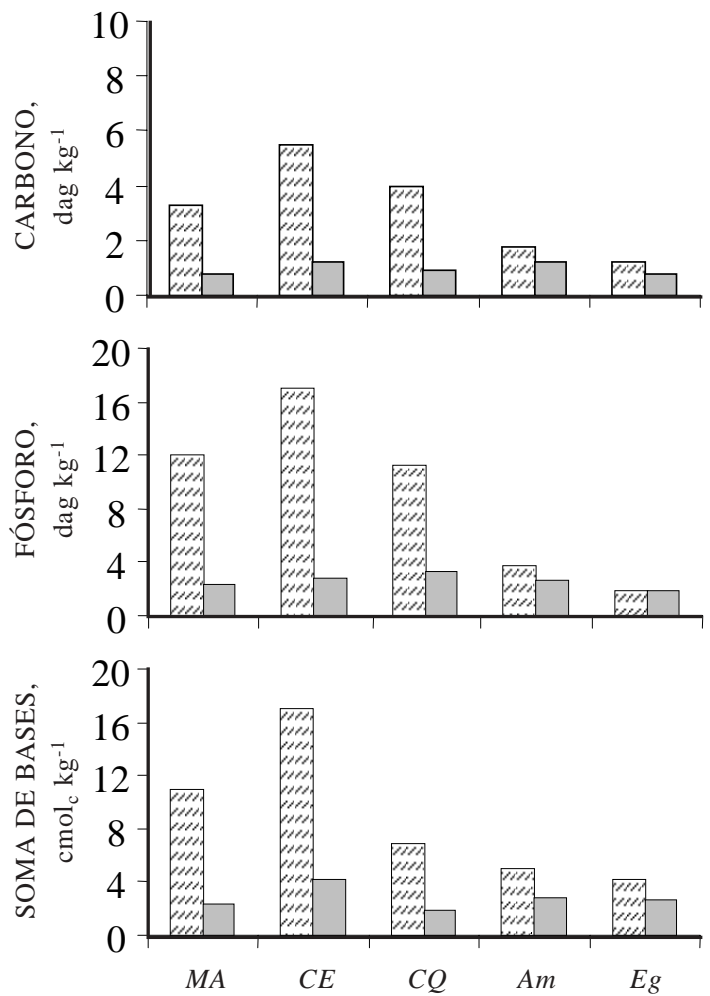
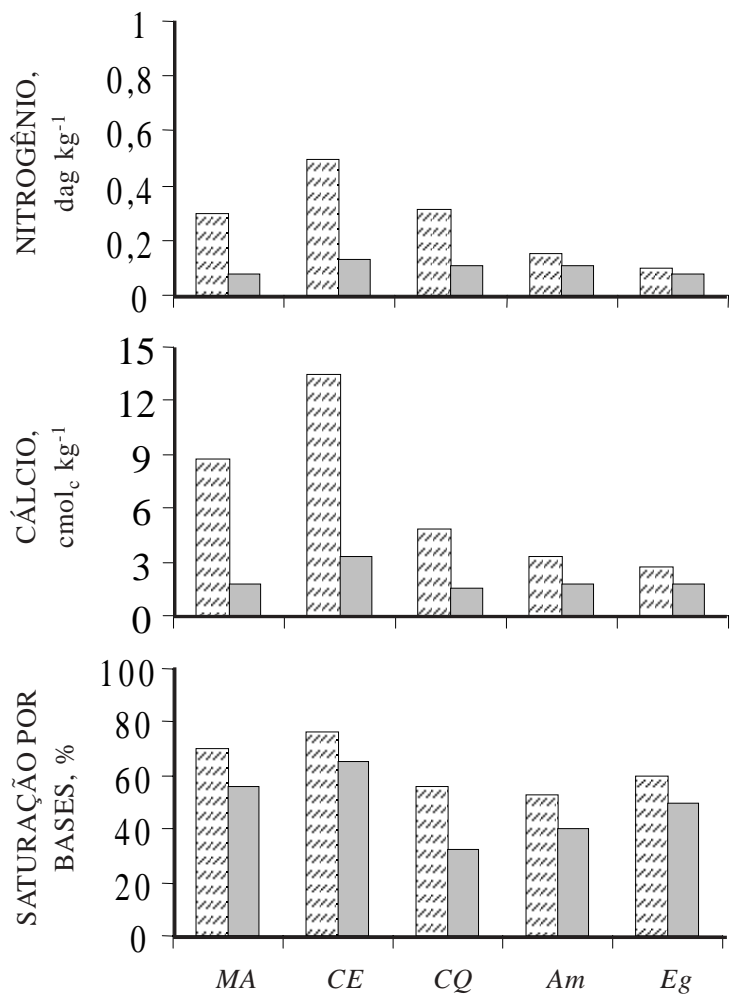

Figura 2. Teor de carbono e nutrientes nos subhorizontes $A_{11}$ (listras) e $A_{12}$ (cinza) em um sistema primário (Mata Alta, MA) e dois secundários (Capoeira de Extração, CE, e Capoeira Quei mada, CQ) de Mata Atlântica de Tabuleiros e nas plantações de Acacia mangium (Am) e Eucalyptus grandis (E g). Nos sistemas florestais nativos, o subhorizonte $A_{11}$ tem de 0 a $3 \mathrm{~cm}$ e nas plantações de 0 a $6 \mathrm{~cm}$ de espessura. Os dados referentes às áreas de floresta encontram-se publicados em Kindel et al. (1999) e Kindel \& Garay (2001). 
As plantações de A. mangium e E. grandis apresentaram essa mesma tendência (Quadro 2 e Figura 2); no entanto, as diferenças entre as duas profundidades, apesar de significativas estatisticamente, não foram tão acentuadas como nas florestas estudadas por aqueles autores. Analisando as plantações, verificou-seque os teores de $\mathrm{C}$ e $\mathrm{N}$ foram sequer $50 \%$ maiores no subhorizonte $A_{11}$, enquanto o teor de P e a SB nunca chegaram a ser duas vezes maiores no subhorizonte $A_{11}$. As diferenças entre os subhorizontes podem ser atribuídas à incorporação de matéria orgânica nos primeiros centímetros do solo, em decorrência do preparo do solo pelo arado, e, posteriormente, acentuadas pel a ação dos plantios.

Desta forma, pode-se afirmar que, após sete anos do plantio, os teores de carbono e nutrientes do subhorizonte $A_{11}$ nas plantações não seigualaram aos das matas nativas, floresta primária e secundária, originárias de diferentes intervenções e em recuperação por mais de 40 anos, indicando que nos plantios a fertilidade do sol o superficial ainda está aquém do esperado para os sistemas nativos (F igura 2).

\section{CONCLUSÕES}

1. A maior quantidade de matéria orgânica incorporada ao solo pela decomposição em A. mangium foi decorrente da maior qualidade nutricional do fol hiço de acácia e representada pela menor relação $\mathrm{C} / \mathrm{N}$.

2. A maior quantidade de material foliar acumulada nas camadas holorgânicas na área sob A. mangium, o maior teor e estoque de nitrogênio no fol hiço, a menor relação C/N do fol hiço e a maior quantidade de matéria orgânica incorporada ao solo pela decomposição em $A$. mangium refletiram a grande contribuição em matéria orgânica e nitrogênio que esta espécie fornece ao solo, evidenciando sua maior eficiência em reconstituir os horizontes orgânicos do solo.

3. Os maiores teores de carbono, nitrogênio e nutrientes no horizonte $\mathrm{A}$ em $\mathrm{A}$. mangium e a alta correlação que el es apresentaram demonstraram a dependência da fertilidade do sol oà matéria orgânica incorporada.

4. Em relação à matéria orgânica e aos demais atributos do solo, no subhorizonte $A_{11}$ das plantações, não foram encontrados os mesmos teores obtidos na mata nativa, indicando que sete anos de plantio não foram suficientes para restabelecer a fertilidade do solo superficial.

\section{AGRADE CIMENTOS}

Ao Dr. Renato Moraes de J esus, diretor da Reserva Natural da Vale do Rio Doce, pelo apoio e por ceder os dados para a descri ção do método e os de clima. Às entidades financiadoras. À Dra. Aglai da Silva e ao Engenheiro J. Ventosilla, pela ajuda no trabalho decampo. A Roseli Pellens, pela colaboração notrata-mento estatístico dos dados. A Daniel Vidal Perez, da EMBRAPA Solos, pelo esclarecimento de dúvidas.

\section{LITERATURA CITADA}

BABEL, U. Micromorphology of soil organic matter. In: GIESEKING, J .E, ed. Soil component. Berlin, SpringerVerlag, 1975. p.369-473.

BERNHARD-REVERSAT, F. Dynamics of litter and organic matter at the soil-litter interface in fast-growing tree plantations on sandy ferrallitic soils (Congo). Acta Oecol., 14:179-195, 1993.

BERTHELIN, J.; LEYVAL, C. \& TOUTAIN, F. Biologie des sols: Rôle des organismes dans l'alteration et I'humification. In: BONNEAU, M. \& SOUCHIER, B., eds. Pédologie. 2 Constituans et propiétés du sol. Paris, Masson, 1994. p.143211.

EMPRESA BRASILEIRA DE PESQUISA AGROPECUÁRIA EMBRAPA. Manual de métodos de análise de solos. Rio de J aneiro, 1979. 255p.

FRANCO, A.A.; DIAS, L.E.; FARIA, S.M.; CAMPELLO, E.F.C. \& SILVA, E.M.R. Uso deleguminosas florestais noduladas e micorrizadas como agentes de recuperação e manutenção da vida do solo: um model o tecnológico. In: ESTEVES, F., ed. Oecologica Brasiliensis: estrutura, funcionamento e manejo de ecossistemas. Rio de J aneiro, 1995. p.459-467.

GAMA-RODRIGUES, A.C.; BARROS, N.F. \& MENDONÇA, E.S. Alterações edáficas sob plantios puros e misto de espécies florestais nativas do sudeste da Bahia, Brasil. R. Bras. Ci. Solo, 23:581-592, 1999.

GARAY, I.; KINDEL, A.; CALLIPO, A.; BARROS, M.E.O. \& JESUS, R.M. Formas de húmus em ecossistemas de Floresta Costeira Intertropical. I. A Mata Atlântica de Tabuleiros. In: ESTEVES, F., ed. Oecologica Brasiliensis: estrutura, funcionamento e manejo de ecossistemas. Rio de J aneiro, 1995a. p.1-18.

GARAY, I.; KINDEL, A. \& J ESUS, R.M. Diversity of humus forms in the Atlantic F orest ecosystems (Brazil). TheTable Iand Atlantic Forest. Acta Oecol., 16:553-570, 1995b.

GARAY, I. \& KINDEL, A. Diversidade funcional em fragmentos deFloresta Atlântica. Valor indicador das formas dehúmus florestais. In: GARAY, I \& \& DIAS, B., orgs. Conservação da biodiversidade em ecossistemas tropicais: avanços conceituais e revisão de novas metodologias de avaliação e monitoramento. Petrópolis, Vozes, 2001. p.350-368.

HIGA, A.R. \& HIGA, R.C.V. Indicações de espécies para o reflorestamento. In: GALVÃO, A.P.M., org. Reflorestamento de propriedades rurais para fins produtivos e ambientais. Um guia para ações municipais e regionais. Brasília: Embrapa Comunicação para Transferência de Tecnologia e Colombo/PR: Embrapa Florestas, 2000. p.101-124.

KINDEL, A.; BARBOSA, P.M.S.; PÉREZ, D.V. \& GARAY, I. Efeito do extrativismo seletivo de espécies arbóreas da Floresta Atlântica de Tabuleiros na matéria orgânica e outros atributos do solo. R. Bras. Ci. Solo, 23:465-474, 1999. 
KINDEL, A. \& GARAY, I. Caracterização de ecossistemas da Floresta Atlântica de Tabuleiros por meio das formas de húmus. R. Bras. Ci. Solo, 25:551-563, 2001.

KINDEL, A. \& GARAY, I. Humus form in ecosystems of the Atlantic Forest, Brazil. Geoderma, 108:101-118, 2002.

LIMA, W.P. O reflorestamento com eucalipto e seus impactos ambientais. São Paulo, Artpress, 1987. 114p.

LOUZADA, M.A.P.; CURVELLO, A.; BARBOSA, J.H.C. \& GARAY, I. O aporte de matéria orgânica ao solo: quantificação, fenologia e suas relações com a composição específica em área de floresta Atlântica de Tabuleiros. Leandra, 12:27-32, 1997.
MEIS, M.R.M. Contribuição ao estudo do Terciário Superior e Quaternário da baixada da Guanabara. Rio de J aneiro, Universidade Federal do Rio de J aneiro, 1976. (Tese de Doutorado)

PELLENS, R. \& GARAY, I. A comunidade de macroartrópodos edáficos em uma plantação de Coffea robusta Linden (Rubiaceaae) e em uma floresta primária em Linhares, Espírito Santo, Brasil. R. Bras. Zool., 16:245-258, 1999.

RIZZINI, C.M.; ADUAN, R.E.; JESUS, R.M. \& GARAY, I. Contribuição ao conhecimento da Floresta Pluvial de Tabuleiros, Linhares, ES, Brasil. Leandra, 12:54-76, 1997.

SWIFT, M.J .; HEAL, O.W. \& ANDERSON, J .M. Decomposition in terrestrial ecosystems. Oxford, Blackwell Scientific Publications, 1979. 372p. 